\title{
Atividade física na saúde e qualidade de vida de mulheres climatéricas
}

\author{
Physical activity in health and quality of life of climacteric women
}

\author{
Hellen Viviany de Alencar Barreto', Talita Tamara Matos Alves', Nathanael Ibsen da Silva Soares', \\ Vernon Furtado da Silva², Patrícia Uchôa Leitão Cabral' \\ 'Universidade Estadual do Piauí (UESPI), Departamento de Educação Física, Teresina, PI, Brasil. \\ ZUniversidade do Estado do Rio de Janeiro (UERJ), Instituto de Educação Física e Desportos, Rio de Janeiro, RJ, Brasil.
}

Recebido em: julho 2015 / aceito em: setembro 2015

naelibsen@hotmail.com

\section{RESUMO}

Objetivo: avaliar a saúde e qualidade de vida (OV) de mulheres praticantes e não praticantes de exercícios físicos (EF). Método: estudo transversal que compreendeu 60 mulheres entre 40 a 60 anos, sendo 30 praticantes de EF e 30 não praticantes. Utilizou-se dois questionários, sendo um inerente às características sociodemográficas e clínicas, e o Women's Health Questionnarie (WHQ), que permite avaliar a saúde e a qualidade de vida de mulheres no período do climatério. Resultados: as mulheres praticantes de EF apresentaram menor sintomatologia climatérica e melhor estado de saúde que as mulheres não praticantes. Observou-se que dentre os domínios do WHQ, a depressão, os sintomas somáticos, os vasomotores, a ansiedade, os problemas com o sono e a memória/concentração foram os que mostraram diferenças significativas $(p<0,05)$ entre os grupos. Considerações finais: a prática atividades físicas parece contribuir positivamente para a saúde e a qualidade de vida de mulheres no climatério.

Palavras-chave: Climatério; Mulheres; Atividade física.

\section{ABSTRACT}

Objective: to evaluate the health and quality of life (OOL) of women practitioners and not practitioners physical exercise (PE). Method: cross-sectional study comprised 60 women aged $40-60$ years, being 30 $P E$ practitioners and 30 non-practicing. Two questionnaires were used, one inherent to sociodemographic and clinical characteristics, and the Women's Health Questionnaire (WHQ), being this one used to evaluate the health and quality of life of women during the climacteric period. Results: women practitioners of EF had lower climacteric symptoms and better health than non-practicing women. It was observed that among the factors of the WHO the ones related to depression, somatic symptoms, vasomotor symptoms, anxiety, sleep problems, and the memory/concentration showed significant differences $(p<0.05)$ between groups. Closing remarks: that physical activity appears to contribute positively to the health and quality of life in climacteric women.

Keywords: Climacteric; Women; Physical activity.

\section{INTRODUÇ̃̃O}

Atualmente, o interesse pelo estudo da qualidade de vida de mulheres durante o climatério tem sido crescente, devido em grande parte ao aumento da expectativa de vida feminina. ${ }^{1}$ E neste sentido, é possível prever que este grupo possa viver um terço de suas vidas após a menopausa. ${ }^{2}$

A Qualidade de Vida (OV) é referida pela Organização Mundial de Saúde (OMS) como a percepção que o indivíduo tem de sua posição na vida, no contexto da cultura e dos sistemas de valores em que vive, com base em suas metas, objetivos, expectativas e preocupações pessoais. ${ }^{3}$ A QV faz parte de um constructo multidimensional que envolve as dimensões psíquicas, sociais, físicas e emocionais. ${ }^{4}$

Segundo Lorenzi et al. ${ }^{5}$ os fatores mais relevantes associados à qualidade de vida no climatério são aqueles ligados às condições físicas e emocionais prévias, assim como a inserção social e experiências frente a eventos vitais. Hoje, tem-se reconhecido a influência das atitudes e percepções da mulher em relação à menopausa na qualidade de vida durante o climatério..$^{6,4}$ 
Observa-se que mulheres com uma percepção mais negativa da menopausa e do envelhecimento não somente tendem a apresentar uma pior qualidade de vida, como sintomas climatéricos mais severos. ${ }^{7}$

No climatério, com hipoestrogenismo progressivo, a maioria das mulheres refere o aumento da incidência de sintomas psicológicos, somáticos, vasomotores e urogenitais, nos quais tendem a influenciar negativamente a qualidade de vida. ${ }^{8}$ Alguns dos sintomas mais comuns nessa fase são os fogachos (ondas de calor), palpitações, tonturas, cansaço, cefaléia, memória fraca, insônia, dores articulares, ansiedade, irritabilidade, depressão, pele ressecada, maior disposição de gordura abdominal, secura vaginal e urgência miccional. ${ }^{9,10}$

A preocupação com a qualidade de vida em saúde ao longo do processo de envelhecimento tem aumentado exponencialmente nos últimos anos, porém a qualidade de vida no climatério infelizmente ainda é pouco explorada no Brasil. ${ }^{10}$ Diversos instrumentos para avaliação da qualidade de vida relacionada ao climatério têm sido construídos desde 1970. Dentre esses instrumentos o Women's Health Questionnaire (WHQ) tem sido bastante utilizado por aferir medidas específicas populacionais do climatério. Considera-se que a qualidade da saúde relativa à menopausa é tão melhor quanto menor a pontuação atribuída pela paciente às dimensões descritas no WHO. ${ }^{11}$

Uma das alternativas não farmacológicas mais eficazes na redução dos sintomas climatéricos e na prevenção primária e secundária de inúmeras doenças crônicas é a prática de exercícios físicos (EF).12 Estudos mostram que a sintomatologia climatérica apresenta-se menos intensa entre as mulheres fisicamente ativas, quando comparadas às menos ativas e/ou sedentárias. ${ }^{13,14}$

Tairova e De Lorenzi ${ }^{15}$ mostraram em seu estudo que as mulheres de meia-idade, fisicamente ativas, apresentaram maior qualidade de vida nos domínios somato-vegetativos, urogenitais e psicológicos, quando comparados às mulheres sedentárias. Pesquisa de base populacional no nordeste brasileiro também mostrou que as mulheres climatéricas com maior nível de atividade física tinham maiores escores de qualidade de vida em comparação com as mulheres sedentárias. ${ }^{16}$

Nesse contexto a prática regular de exercícios físicos parece ser uma opção terapêutica eficaz para a melhora da qualidade de vida de mulheres no período do climatério. ${ }^{7,17}$ Porém, ainda existe uma necessidade eminente de realização de mais estudos que divulguem a importância desse tema. O presente estudo propõe avaliar a saúde e qualidade de vida de mulheres praticantes e não praticantes de exercícios físicos regulares.

\section{MÉTODO}

Foi realizado um estudo descritivo de corte transversal que compreendeu a população feminina com idade entre 40 e 60 anos. Utilizou-se o processo de amostragem não probabilística com seleção por conveniência das participantes, a qual resultou em 60 mulheres, sendo que 30 destas eram praticantes de exercícios físicos supervisionados no parque municipal Nova Potycabana (grupo praticante) e 30 não praticavam exercícios físicos regulares (grupo não praticante).
As mulheres não praticantes de exercício físico foram recrutadas do grupo da pastoral familiar. A pesquisa foi realizada entre os meses de Agosto e Outubro de 2013, na cidade de Teresina.

Foram considerados critérios de inclusão para o grupo das mulheres praticantes de EF, ter a idade compreendida entre 40 a 60 anos e serem praticantes de qualquer modalidade de exercício físico com assiduidade de pelo menos 3 vezes na semana por no mínimo três meses. Para o grupo das mulheres não praticantes de $E F$, foram determinados que as mesmas não devessem estar realizando nenhum tipo de exercício físico por pelo menos três meses, tempo no qual todas as adaptações do treinamento anterior são perdidas. ${ }^{18}$

Foram considerados como critérios de exclusão: uso de terapia hormonal, hormonioterapia por implantes, assim como a incapacidade da mulher em participar do estudo, ou qualquer fator que impedisse a entrevista (falta de tempo, analfabetismo), bem como a recusa explícita em participar do estudo.

Todas as participantes, após terem sido devidamente esclarecidas sobre a proposta do estudo e procedimentos aos quais seriam submetidas, assinaram um termo de consentimento livre e esclarecido. Em seguida responderam ao questionário que compreendia as características sociodemográficas e clínicas, e posteriormente o Women's Health Questionnarie (WHO).

O Women's Health Questionnarie (WHQ) permite avaliar a saúde e a qualidade de vida de mulheres no climatério. $O$ instrumento avalia mudanças físicas e aspectos relacionados à saúde e o bem estar de mulheres nesse período da vida. É composto por 37 questões, sendo 01 subjetiva e 36 objetivas, com quatro alternativas de resposta. A questão subjetiva aborda a dificuldade que a mulher possa ter em lidar com alguns dos sintomas apresentados no instrumento. As questões objetivas são divididas em nove domínios, dispostos aleatoriamente, que avaliam: depressão (tristeza, perda de interesse por aspectos da vida, perda de apetite, irritabilidade, nível de sensação de bem estar - questões: $3,5,7,8,10,12,25$ ); sintomas somáticos (dores de cabeça, cansaço físico, tontura, dores nas costas e nos membros, náuseas/mal estar, formigamento nas mãos/ pés, urgência urinária e sede constante - questões: 14, $15,16,18,23,30,35)$; memória e concentração (humor, dificuldade de concentração, memória - questões: 20, 33, 36); sintomas vasomotores (fogachos e suores noturnos - questões: 19 e 27); ansiedade/temores (medo/pânico, ansiedade, palpitações/aperto no peito, tensão/nervosismo - questões: 2, 4, 6, 9); comportamento sexual (libido, satisfação com vida sexual, secura vaginal - questões: $24,31,34$ ); problemas de sono (sono irregular, impaciência, sonolência - questões: 1, 11, 29); sintomas menstruais (dores nos seios, cólicas, hemorragias, sensação de mal estar questões: 17, 22, 26, 28); e atratividade (preocupação com o envelhecimento, energia física e mental, sensação de atratividade física questões: $13 ; 21 ; 32$ ). Quanto maior o valor dos escores obtidos no WHQ, menor a gravidade dos sintomas, melhor o estado de saúde e qualidade de vida das mulheres. ${ }^{19}$

O processamento dos dados e a análise estatística foram realizados por meio do programa Statistical Package for the Social Science (SPSS), versão 18.0. Primeiramente foi aplicado o teste de Shapiro-Wilk 
para avaliar a normalidade das variáveis quantitativas. Como as variáveis não apresentaram distribuição normal aplicou-se testes não paramétricos As variáveis quantitativas foram apresentadas por meio de média e desvio padrão. Para verificar diferenças entre duas médias aplicou-se teste Mann-Whitney e para verificar associação entre as variáveis qualitativas utilizou-se o teste qui-quadrado de Pearson $\left(\chi^{2}\right)$. Considerou-se estatisticamente significativo um valor de $p<0,05$.

Este estudo foi aprovado pelo Comitê de Ética sob o parecer de $n^{\circ} 785.261$, obedecendo aos padrões éticos de acordo com as normas da Resolução 466/12 do Conselho Nacional de Saúde para pesquisas com seres humanos do Ministério da Saúde.

\section{RESULTADOS}

As características sociodemográficas e clínicas da amostra estão demostradas na tabela 1. Observa-se que não houve diferenças significativas $(p<0,05)$ entre o grupo de mulheres praticantes e não praticantes de exercícios físicos.
As mulheres praticantes de exercício físico apresentaram escores mais elevados em todos os aspectos físicos e de bem estar abordados no WHQ, quando comparadas com as mulheres que não praticam nenhum exercício físico (Tabela 2). De acordo com o instrumento proposto quanto maior os escores, melhor a qualidade de vida relacionada à saúde da mulher no climatério. Observa-se que a depressão, os sintomas somáticos, os sintomas vasomotores, a ansiedade, os problemas com o sono e a memória/concentração mostraram diferenças significativas $(p<0,05)$ entre os dois grupos avaliados.

A Figura 1 mostra que $75 \%$ das mulheres não têm dificuldades em lidar os sintomas relatados no WHO. Dentre os sintomas, os mais relatados pelas mulheres foram: dores nas costas e membros $(8,3 \%)$, as dores de cabeça $(3,3 \%)$, os fogachos $(3,3 \%)$, a impaciência $(3,3 \%)$.

\section{DISCUSSÃO}

Não foi observada diferença significativa entre os grupos com relação às características sociodemográficas e comportamentais.

Tabela 1 - Perfil sociodemográfico e comportamental das mulheres praticantes e não praticantes de exercícios físicos regulares $(n=60)$.

\begin{tabular}{|c|c|c|c|c|c|}
\hline & \multicolumn{2}{|c|}{ Grupo praticante $(n=30)$} & \multicolumn{2}{|c|}{ Grupo não praticante $(n=30)$} & \multirow[b]{2}{*}{$P$} \\
\hline & $\mathbf{N}$ & $\%$ & $\mathbf{N}$ & $\%$ & \\
\hline Faixa etária & & & & & $0,795^{\mathrm{a}}$ \\
\hline 40-50 anos & 13 & 43,3 & 14 & 46,7 & \\
\hline $50-60$ anos & 17 & 56,7 & 16 & 53,3 & \\
\hline Média (D.P) & \multicolumn{2}{|c|}{$49,8(6,6)$} & \multicolumn{2}{|c|}{$49,7(5,1)$} & $0,965^{b}$ \\
\hline Raça & & & & & $1,000^{\mathrm{a}}$ \\
\hline Branca & 06 & 20,0 & 06 & 20,0 & \\
\hline Não branca & 24 & 80,0 & 24 & 80,0 & \\
\hline Estado civil & & & & & $0,920^{a}$ \\
\hline Solteira & 04 & 13,3 & 05 & 16,7 & \\
\hline Casada/União estável & 16 & 53,3 & 18 & 60,0 & \\
\hline Viúva & 03 & 10,0 & 02 & 6,7 & \\
\hline Separada & 02 & 6,7 & 02 & 6,7 & \\
\hline Divorciada & 05 & 16,7 & 03 & 10,0 & \\
\hline Escolaridade & & & & & $0,939^{a}$ \\
\hline Fund. Incompleto & 05 & 16,7 & 05 & 16,7 & \\
\hline Fund. Completo & 01 & 3,3 & 0 & 0,0 & \\
\hline Médio Incompleto & 01 & 3,3 & 01 & 3,3 & \\
\hline Médio Completo & 12 & 40,0 & 14 & 46,7 & \\
\hline Superior Incompleto & 02 & 6,7 & 02 & 6,7 & \\
\hline Superior Completo & 08 & 26,7 & 06 & 20,0 & \\
\hline Pós-graduação & 01 & 3,3 & 02 & 6,7 & \\
\hline Renda familiar & & & & & $0,740^{\mathrm{a}}$ \\
\hline$<1 \mathrm{SM}$ & 02 & 6,7 & 03 & 10,0 & \\
\hline 1 a 2 SM & 14 & 46,7 & 10 & 33,3 & \\
\hline 2 a 4 SM & 05 & 16,7 & 07 & 23,3 & \\
\hline$\geq 4 \mathrm{SM}$ & 09 & 30,0 & 10 & 33,3 & \\
\hline Menopausa & & & & & $0,438^{a}$ \\
\hline Sim & 14 & 46,7 & 17 & 56,7 & \\
\hline Não & 16 & 53,3 & 13 & 43,3 & \\
\hline Média (D.P) & \multicolumn{2}{|c|}{$6,9(6,6)$} & \multicolumn{2}{|c|}{$5,6(5,5)$} & $0,575^{b}$ \\
\hline Estado nutricional-IMC $\left(\mathrm{kg} / \mathrm{m}^{2}\right)$ & & & & & $0,175^{a}$ \\
\hline Eutrófico & 17 & 56,7 & 12 & 40,0 & \\
\hline Sobrepeso & 09 & 30,0 & 16 & 53,3 & \\
\hline Obesidade & 04 & 13,3 & 02 & 6,7 & \\
\hline Média (D.P) & \multicolumn{2}{|c|}{$25,1(3,4)$} & \multicolumn{2}{|c|}{$25,6(3,4)$} & $0,568^{b}$ \\
\hline
\end{tabular}

SM: Salários Mínimos (R\$: 678,00); D.P: Desvio padrão - variância em torno da média.

Testes estatísticos: a: Teste qui-quadrado de Pearson $\left(\mathbf{\square}^{2}\right)$; b: Teste Mann-Whitney. 
Tabela 2 - Escores dos domínios do Women's Health Questionnaire (WHQ) de mulheres praticantes e não praticantes de exercício físico.

\begin{tabular}{|c|c|c|c|c|c|}
\hline \multirow[t]{2}{*}{ WHQ } & \multicolumn{2}{|c|}{ Grupo Praticante $(n=30)$} & \multicolumn{2}{|c|}{ Grupo não praticante $(n=30)$} & \multirow[b]{2}{*}{$P^{*}$} \\
\hline & Média & D.P & Média & D.P & \\
\hline Depressão & 25,5 & 2,9 & 21,8 & 3,7 & $<0,001$ \\
\hline Sintomas somáticos & 21,9 & 4,2 & 17,8 & 3,6 & $<0,001$ \\
\hline Sintomas vasomotores & 6,2 & 1,6 & 5,0 & 1,5 & 0,003 \\
\hline Ansiedade/Tremores & 13,8 & 2,5 & 11,3 & 3,5 & 0,003 \\
\hline Comportamento sexual & 9,2 & 3,1 & 8,5 & 3,3 & 0,334 \\
\hline Problemas no sono & 9,4 & 1,9 & 8,0 & 2,3 & 0,021 \\
\hline Sintomas menstruais & 13,5 & 2,4 & 12,6 & 3,2 & 0,230 \\
\hline Atratividade & 10,4 & 1,6 & 9,8 & 2,0 & 0,210 \\
\hline Memória e concentração & 9,0 & 2,2 & 7,2 & 2,1 & 0,004 \\
\hline
\end{tabular}

*Teste Mann-Whitney

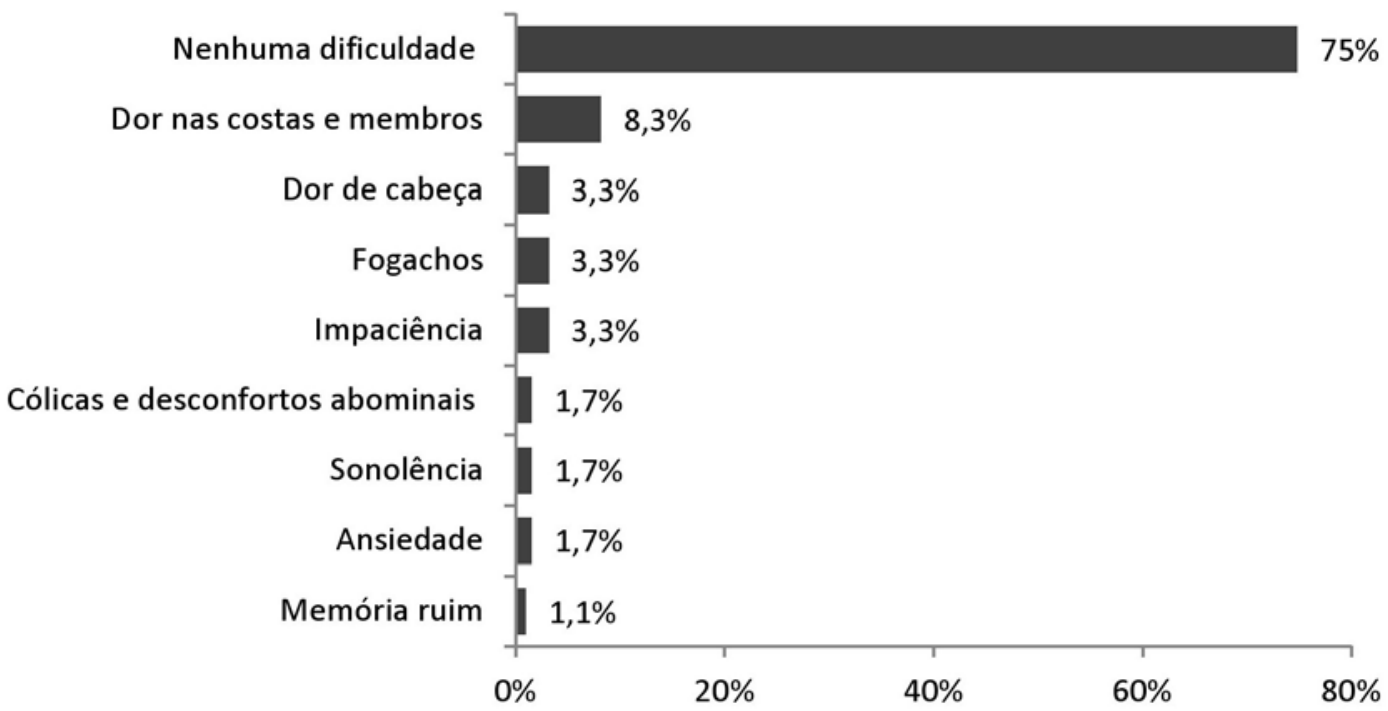

Figura 1 - Dificuldades relatadas pelas mulheres para lidar com os sintomas do climatério, em função da pergunta subjetiva: qual a dificuldade em lidar com alguns dos sintomas apresentados no instrumento do questionário (WHQ)?

No presente estudo, observou-se que a prática de exercícios físicos regulares parece influenciar positivamente a saúde física e mental das mulheres no período do climatério. As mulheres praticantes de EF apresentaram menor intensidade em todos os sintomas climatéricos quando comparado às mulheres não praticantes de exercícios físicos. A depressão, os sintomas somáticos, os sintomas vasomotores, a ansiedade, os problemas com o sono e a memória/concentração, mostraram diferença significativa entre os grupos, visto que o grupo das mulheres não praticantes de EF apresentou piores escores (maior sintomatologia climatérica) que o grupo das mulheres praticantes de exercício físico.

A qualidade de vida é dependente de diversos fatores e por isso, é possível que outras variáveis, como por exemplo, a autoeficácia, que é definida como a crença do indivíduo sobre sua capacidade de desempenhar tarefas para produzir um resultado desejado, possa influenciar na percepção de saúde, condição física e vitalidade de indivíduos, assim como na prática de exercícios físicos. ${ }^{20}$

Lorenzi et al. ${ }^{4}$ em seu estudo mostrou por meio também do WHQ, que a atividade física regular se associou a melhores escores de qualidade vida. Daley et al. ${ }^{21}$ observaram que as mulheres regularmente ativas apre- sentaram melhor qualidade de vida relacionada à saúde que as mulheres que não eram regularmente ativas.

Estudo realizado em Natal - RN, envolvendo 370 mulheres mostrou maior prevalência de nível mais elevado de qualidade de vida no grupo de mulheres mais ativas quando comparadas às sedentárias, correspondendo a uma chance 2,6 vezes maior de melhor qualidade de vida entre as mulheres mais ativas. ${ }^{22}$

Diversos estudos mostraram que os sintomas climatéricos se apresentaram significativamente menos intensos no grupo de mulheres ativas quando comparados com as menos ativas, o que reforça o papel positivo do exercício físico no período do climatério. ${ }^{13-15,22}$

As mulheres praticantes de exercícios físicos, em nosso estudo, apresentaram menos sintomas somáti$\cos$ e vasomotores que as mulheres não praticantes. A esse respeito, pesquisas têm mostrado resultados semelhantes, visto que mulheres com prática regular de exercícios físicos manifestaram menor intensidade de sintomas somáticos, alívio nas ondas de calor e fogachos, melhora do humor e da depressão. ${ }^{18,22,23,24}$

Em mulheres menopausadas a atividade física está associada à saúde psicossocial. Observou em nosso estudo diferença significativa no domínio depressão, visto que as mulheres praticantes de EF 
mostraram melhores resultados que as mulheres não praticantes de exercício físico. Estudo recente realizado com 2204 mulheres comparando as mulheres menos ativas com as mais ativas, observou que a prática de atividades físicas esteve relacionada com a melhora dos sintomas climatéricos, dentre eles os de natura psicológica. ${ }^{14}$ De acordo com Asbury, Chandrruangphen e Collins ${ }^{25}$ a prática de atividade física minimizou os sintomas de depressão e estresse, melhorando consequentemente a qualidade de vida de sua amostra.

Segundo De Lorenzi ${ }^{7}$ os sintomas vasomotores, como as ondas de calor e sudorese, bem como aqueles relacionados à atrofia urogenital parecem ser os únicos sintomas diretamente associados com a queda estrogênica, ao passo que as demais queixas climatéricas (ex. labilidade emocional, menor libido e insônia) estariam relacionadas principalmente ao modo como a menopausa é percebida e ao estilo de vida. Nossos resultados mostraram que as mulheres praticantes de EF apresentaram menor intensidade de sintomas vasomotores e menor problema com o sono que as mulheres não praticantes de exercício físico.

Dentre os sintomas mais difíceis de lidar, segundo o relato das mulheres avaliadas neste estudo, as dores nas costas e nos membros, seguido por dor de cabeça, fogachos e impaciência, obtiveram maior prevalência, no entanto a maioria afirmou não ter dificuldade. Estudos mostram que mulheres com uma percepção mais negativa da menopausa não somente tendem a apresentar uma pior qualidade de vida, como sintomas climatéricos mais severos. ${ }^{7,10} \mathrm{Em}$ seus estudos Mirzaiinjmabadi, Anderson e Barnes ${ }^{17}$ mostraram que a prática de exercício físico regular mostrou ser eficaz no alívio desses sintomas relatados, promovendo assim um meIhor estado de saúde, bem estar e melhor percepção de qualidade de vida.

Importantes estudos aqui relatados têm demons trado os inúmeros benefícios da prática de atividade física regulares. Kakkar et al. ${ }^{26}$ afirmaram que a prática de atividade física regular, ainda que de pouca intensidade, contribuiu globalmente para uma melhoria das condições de saúde, tendo um impacto positivo também na sua esfera psíquica e em muitos dos sintomas característicos do climatério, melhorando, inclusive, os sentimentos e as atitudes das mulheres nessa fase.

Devido ao tipo de delineamento empregado, nossos achados não devem ser generalizados. Importante ressaltar que instrumentos específicos para a avaliação da qualidade de vida como o WHQ, contribuem para um maior aprofundamento do impacto dos sintomas da menopausa e a sua relação com a qualidade de vida. ${ }^{27}$

Outras pesquisas sobre esse tema são necessárias, preferencialmente as prospectivas, de base populacional, com avaliações antes e depois de intervenções de atividades físicas, pois podem oferecer novos horizontes sobre a relação existente entre a prática de atividades físicas, saúde e bem estar de mulheres climatéricas.

\section{CONSIDERAÇÕES FINAIS}

O presente estudo mostrou que os sintomas cli- matéricos são menos intensos entre as mulheres que praticam exercícios físicos quando comparado às muIheres que não praticam nenhum tipo de exercício físico. A prática regular de exercícios físicos esteve associada a uma melhor percepção de saúde e qualidade de vida entre as mulheres no período do climatério.

\section{REFERÊNCIAS}

1. Ministério da Saúde (BR), Secretaria Executiva, Subsecretaria de Planejamento e Orçamento. Sistema de Planejamento do SUS: Uma construção coletiva: Plano Nacional de Saúde (PNS) 2008/2009-2011. Brasília (DF): Ministério da Saúde; 2010.

2. Spritzer PM, Wender MCO. Terapia hormonal na menopausa: quando não usar. Arq Bras Endocrinol Metab 2007; 51(7): 1058-63.

3. WHOQOL Group. Study protocol for the World Health Organization project to develop a quality of life assessment instrument (WHOQOL). Qual Life Res 1993; 2: 153-159.

4. De Lorenzi DRS, Baracat EC, Saciloto B, Padilha Júnior I. Fatores associados à qualidade de vida após menopausa. Rev Assoc Med Bras 2006; 52(5): 312-7.

5. De Lorenzi DRS, Catan LB, Moreira K, Ártico GR. Assistência à mulher climatérica: novos paradigmas. Rev bras enferm. 2009; 62(2): 287-93.

6. Zahar SEV, Aldrighi JM, Tostes MA, Russomano F, Zahar LO. Avaliação da qualidade de vida na menopausa. Reprod Clim 2001; 16(3): 163-72.

7. De Lorenzi DRS, Danelon C, Saciloto B, Padilha Junior I. Fatores indicadores da sintomatologia climatérica. Rev Bras Ginecol Obstet 2005; 27(1): 7-11.

8. Blümel JE, Chedraui $P$, Baron G, Belzares E, Bencosme A, Calle $A$, et al. A large multinational study of vasomotor symptom prevalence, duration, and impact on quality of life in middle-aged women. Menopause 2011; 18(7): 778-85.

9. Bulcão CB, Carange $E$, Carvalho HP, Ferreira-França JB, Kligerman-Antunes J, Backkes J, et al. Aspectos fisiológicos, cognitivos e psicossociais da senescência sexual. Ciências Cognição 2004; 1(1): 54-75.

10. De Lorenzi DRS. Avaliação da qualidade de vida no climatério. Rev Bras Ginecol Obstet 2008; 30(3): 103-6.

11. Hunter MS. The Women's Health Questionnaire (WHO): Frequently Asked Questions (FAQ). Health Qual Life Outcomes 2003; 1(1): 41.

12. Leitão MB, Lazzoli JK, Oliveira MAB, Nóbrega ACL, Silveira GG, Carvalho T, et al. Posicionamento oficial da Sociedade Brasileira de Medicina do Esporte: atividade física e saúde na mulher. Rev Bras Med Esporte 2000; 6(6): 215-20.

13. Tan MN, Kartal M, Guldal D. The effect of physical activity and body mass index on menopausal symptoms in Turkish women: a cross-sectional study in primary care. BMC Women's Health 2014; 14: 38.

14. Kim MJ, Cho J, Ahn Y, Yim G, Park HY. Association between physical activity and menopausal symptoms in perimenopausal women. BMC Womens Health 2014; 14: 122.

15. Tairova OS, De Lorenzi, DRS. Influência do exercício físico na qualidade de vida de mulheres na pós-menopausa: um estudo caso-controle. Rev bras geriatr gerontol 2011; 14(1): 135-45.

16. Gonçalves AKS, Canário ACG, Cabral PUL, Silva RAH, Spyrdes MHC, Giraldo PC, Eleutério Júnior J. Impacto da atividade física na qualidade de vida de mulheres de meia idade: estudo de base populacional. Rev Bras Ginecol Obstet 2011; 33(12): 408-413.

17. Mirzaiinjmabadi K, Anderson D, Mames M. The relationship 
between exercise, body mass index and menopausa symptoms in midlife Australian women. Int $\mathrm{J}$ Nurs Pract 2006; 12(1): 28-34.

18. Garber CE, Blissmer B, Deschenes MR, Franklin BA, Lamonte MJ, Lee IM et al. American College of Sports Medicine. American College of Sports Medicine position stand. Quantity and quality of exercise for developing and maintaining cardiorespiratory, musculoskeletal, and neuromotor fitness in apparently healthy adults: guidance for prescribing exercise. Med Sci Sports Exerc 2011; 43(7): 1334-59.

19. Dias RDS, Ramos CC, Kerr CF, Trinca LA, Cerqueira A, Dalben I et al. Adaptação para o português do questionário de auto-avaliação de percepção de saúde física e mental da mulher de meia-idade - QSM. Rev Psiq Clín 2002; 29(4): 181-9.

20. Wendel-Vos GC, Schuit AJ, Tijhuis MA, Kromhout D. Leisure time physical activity and health-related quality of life: crosssectional and longitudinal associations. Qual Life Res 2004; 13(3): 667-77.

21. Daley A, Macarthur C, Stokes-Lampard H, McManus R, Wilson S, Mutrie N: Exercise participation, body mass index, and health-related quality of life in women of menopausal age. Br J Gen Pract 2007; 57(535): 130-135.
22. Canário $A C$, Cabral PU, Spyrides $M H$, Giraldo PC, Eleutério $\mathrm{J} J \mathrm{r}$, Gonçalves AK. The impact of physical activity on menopausal symptoms in middle-aged women. Int $\mathrm{J}$ Gynaecol Obstet 2012; 118(1): 34-6.

23. Elavsky S, McAuley E. Physical activity and mental health outcomes during menopause: a randomized controlled trial. Ann Behav Med 2007; 33(2): 132-42.

24. Elavsky S: Physical activity, menopause and quality of life: The role of affect and self-worth across time. Menopause 2009; 16(2): 265-271.

25. Asbury EA, Chandrruangphen $P$, Collins P. The importance of continued exercise participation in quality of life and psychological well-being in previously inactive postmenopausal women: a pilot study. Menopause 2006; 13(4): 561-567.

26. Kakkar V, Kaur D, Chopra K, Kaur A, Kaur IP. Assessment of the variation in menopausal symptoms with age, education and working/non-working status in north-Indian sub population using menopause rating scale (MRS). Maturitas. 2007; 57(3): 306-14.

27. Minayo MCS, Hartz ZMA, Buss PM. Qualidade de vida e saúde: um debate necessário. Cien Saude Coletiva 2000; 5(1): 7-18. 